\title{
A hydrophilic gel matrix for single-molecule super-resolution microscopy
}

\author{
Patrick JM Zessin ${ }^{\dagger}$, Carmen L Krüger ${ }^{\dagger}$, Sebastian Malkusch ${ }^{\dagger}$, Ulrike Endesfelder and Mike Heilemann ${ }^{*}$
}

\begin{abstract}
Background: Novel microscopic techniques which bypass the resolution limit in light microscopy are becoming routinely established today. The higher spatial resolution of super-resolution microscopy techniques demands for precise correction of drift, spectral and spatial offset of images recorded at different axial planes.

Methods: We employ a hydrophilic gel matrix for super-resolution microscopy of cellular structures. The matrix allows distributing fiducial markers in 3D, and using these for drift correction and multi-channel registration. We demonstrate single-molecule super-resolution microscopy with photoswitchable fluorophores at different axial planes. We calculate a correction matrix for each spectral channel, correct for drift, spectral and spatial offset in 3D.

Results and discussion: We demonstrate single-molecule super-resolution microscopy with photoswitchable fluorophores in a hydrophilic gel matrix. We distribute multi-color fiducial markers in the gel matrix and correct for drift and register multiple imaging channels. We perform two-color super-resolution imaging of click-labeled DNA and histone $\mathrm{H} 2 \mathrm{~B}$ in different axial planes, and demonstrate the quality of drift correction and channel registration quantitatively. This approach delivers robust microscopic data which is a prerequisite for data interpretation.
\end{abstract}

Keywords: Single-molecule localization microscopy; Super-resolution microscopy; Drift correction; Registration

\section{Background}

Various techniques that bypass the resolution limit in light microscopy were established for cellular imaging (Galbraith and Galbraith 2011, Heilemann 2010, Hell 2009). These techniques, commonly summarized as super-resolution microscopy, resolve cellular structures up to the near-molecular level and still profit from the advantages of fluorescence microscopy, such as high contrast and live cell compatibility. However, at these small spatial scales, accurate correction for drift and spatial or spectral offsets is required. Single-molecule localization microscopy techniques (Betzig et al. 2006, Folling et al. 2008, Heilemann et al. 2008, Hess et al. 2006, Rust et al. 2006) are particularly prone to drift, as many thousands of images and thus long acquisition times are required.

Drift can be minimized by hardware, e.g. with temperature chambers that enclose the microscope (Adler and Pagakis 2003), devices which uncouple the sample

\footnotetext{
* Correspondence: heilemann@chemie.uni-frankfurt.de

${ }^{\dagger}$ Equal contributors

Institute of Physical and Theoretical Chemistry, Goethe-University Frankfurt, Max-von-Laue-Str. 7, 60438, Frankfurt, Germany
}

from the sample holder (van de Linde et al. 2011) or by implementing feedback loops (Carter et al. 2007, Pertsinidis et al. 2010). Drift was reported to be minimized to $0.64 \mathrm{~nm}$ for an acquisition time of several hours (Pertsinidis et al. 2010). Software-based drift correction can be achieved by image correlation of bright-field (Mennella et al. 2012) or super-resolution images (Huang et al. 2008, Mlodzianoski et al. 2011). This approach works particularly well in the presence of structured or dense features. In a dual-objective configuration, anti-correlated changes of the point-spread function (PSF) were analyzed and used for drift correction (Xu et al. 2012). Alternatively, fiducial markers can be added to the sample (Betzig et al. 2006, Rust et al. 2006), and tracking their position over time can be used to correct for drift. This approach is independent of the structure or density of the sample. In addition, multi-spectral markers offer the additional advantage to correct for chromatic offset and register multicolor super-resolution images (Churchman et al. 2005, Malkusch et al. 2012). Fiducial markers are added to the sample and distributed at the surface of the cover slip. The level of lateral drift can be assessed by the standard deviation of the localization of the markers and were

\section{实}


reported between 8 and $10 \mathrm{~nm}$ (Quan et al. 2010, Rust et al. 2006, Shtengel et al. 2009). Recently, drift correction through image correlation was demonstrated for 3D singlemolecule localization microscopy (McGorthy et al. 2013).

Here, we introduce a simple experimental protocol to distribute fiducial markers in three dimensions using a hydrophilic extracellular matrix (ECM). Such hydrogels were developed for cell culture to support cell growth in 3D and serve as a scaffold (Lee et al. 2008), and comprise biocompatible compounds such as fibrin (Blomback 2004 \#212), collagen (Bell et al. 1979) or alginate (Augst et al. 2006). Adding fiducial markers to the ECM gel and embedding the cells in the gel matrix leads to a distribution of the fiducial markers in 3D. We perform single-molecule localization microscopy of DNA and histone H2B in different axial planes, and use the fiducial markers to correct for both drift and spectral offset. A sufficiently dense distribution of fiducial markers further allows registering super-resolution images recorded in different axial planes.

\section{Materials and methods Cell culture}

HeLa cells (300194, Cell Line Service, Eppelheim, Germany) were seeded into 8 chamber cell culture dishes with coverslip bottom (Sarstedt) and cultured at $37^{\circ} \mathrm{C}, 5 \%$ $\mathrm{CO}_{2}$ in RPMI 1640 (PAA Laboratories GmbH, Pasching, Austria) supplemented with $1 \%$ penicillin-streptomycin (PAA Laboratories $\mathrm{GmbH}$, Germany), $2 \mathrm{mM}$ L-glutamin (PAA Laboratories $\mathrm{GmbH}$ ), 10\% fetal bovine serum (FBS, Gibco/Invitrogen, Grand Island, NY, USA), 1\% nonessential amino acids (PAA Laboratories $\mathrm{GmbH}$ ) and 1 $\mathrm{mM}$ sodium pyruvate (PAA Laboratories $\mathrm{GmbH}$ ).

\section{Cloning and transfection}

$\mathrm{H} 2 \mathrm{~B}$ was fused to mEos2 and cloned into a CMV promotor driven backbone (Clontech C2). 24 hours after seeding, HeLa cells were transiently transfected with this plasmid, using FugeneHD (Promega Corporation, Madison, WI, USA).

\section{DNA labelling}

24 hours after seeding, 5-ethynyl-2'-deoxyuridine (EdU, Invitrogen, Eugene, Oregon, USA) was added to a final concentration of $10 \mu \mathrm{M} .15$ minutes after incubation cells were fixed for 15 minutes with $4 \%$ formaldehyde (FA) solution in phosphate buffered saline (PBS, Sigma) $\mathrm{pH} 7$, which was freshly prepared from $38 \%$ stock solution (Sigma). Subsequently, cells were permeabilized with $0.5 \%$ Triton X-100 (Sigma) and washed with PBS containing 3\% (w/v) BSA (bovine serum albumin, Sigma). Click chemistry labeling of EdU with dye azide was performed in a reaction buffer based on protocol published by $\mathrm{Qu}$ et al. (2011) which was further optimized to preserve fluorescence emission of fluorescence proteins (100 mM HEPES pH 8.2, $1 \mathrm{mM} \mathrm{CuSO}$ 4, $50 \mathrm{mM}$ aminoguanidine and $25 \mathrm{mM}$ ascorbic acid). HeLa cells were incubated in the reaction buffer for 15 minutes, and washed with PBS. Labeling of EdU was performed with either Alexa Fluor 647 solely or Alexa Fluor 647 and Alexa Fluor 488 in equal amounts.

\section{Sample preparation}

Extracellular matrix (ECM) gel from Engelbreth-HolmSwarm murine sarcoma (Sigma, protein concentration 7-9 $\mathrm{mg} / \mathrm{mL}$, index of refraction 1.34) was thawed on ice. Fiducial markers (TetraSpecks, $100 \mathrm{~nm}$, Invitrogen) were sonicated for 1 minute and diluted 1:5 in water. $29 \mu \mathrm{l}$ of the liquid ECM gel and $1 \mu \mathrm{l}$ of the fiducial marker solution were mixed, sonicated and carefully added to the fixed cells and incubated at $37^{\circ} \mathrm{C}$ until solidification ( 1-2 h). The samples were post-fixated with $4 \% \mathrm{FA}$ in PBS for 15 minutes to obtain a stable, temperatureindependent solidification. The sample preparation is illustrated in Additional file 1: Figure S1a.

\section{Single-molecule localization microscopy}

Super-resolution imaging was performed either on a commercial system (N-STORM, Nikon) equipped with a cylindrical lens for 3D imaging and a feedback loop driven axial stabilizing system (Perfect Focus System, Nikon) or a custom-built microscope essentially described earlier (Endesfelder et al. 2013). Dual-color imaging of Alexa Fluor 647 and Alexa Fluor 488 was performed in PBS with $100 \mathrm{mM}$ beta-mercaptoethylamine (MEA, Sigma) added and pH set to 7.5. Dual-color imaging of Alexa Fluor 647 and mEos2 was performed in $0.5 \mathrm{mg} / \mathrm{ml}$ (100 units $/ \mathrm{ml}$ ) glucose oxidase (Sigma), 40 $\mathrm{mg} / \mathrm{ml}$ (2000 units/ml) catalase (Roche Applied Science), $10 \% \mathrm{w} / \mathrm{v}$ glucose and $10 \mathrm{mM} \mathrm{MEA}$ in $\mathrm{PBS}$ at $\mathrm{pH} 8$ (Endesfelder et al. 2011). Imaging in different axial planes was realized with a highly inclined and laminated optical sheet (HILO) illumination mode (Tokunaga et al. 2008). The different spectral channels were recorded sequentially.

\section{Drift correction and image registration}

Single-molecule localization data was analyzed with rapidSTORM (Wolter et al., 2010) or the N-STORM software (STORM plugin, NIS, Nikon). As the fiducial markers are found in a large number of frames, we obtain the coordinates of the markers over time, which we want to call trajectory. The trajectories of fiducial markers were extracted using either the "track emission" filter of rapidSTORM or custom-written software.

Trajectories which did not span sufficient long time periods were rejected. All other trajectories were rendered with a Gaussian low pass filter. Drift correction 
was performed with affine matrices (see Additional file 1: Figure S1b and Additional file 2: Figure S2). The registration of different spectral channels was performed by identifying fiducial markers that were present in both spectral data sets. A non-linear translation matrix was calculated from all fiducial markers in both channels. All coordinate processing routines were written in Python, Scipy and Numpy (Peterson 2009) (Additional file 1: Figure S1b). Super-resolution images were generated with rapidSTORM and overlaid in Fiji (Schindelin et al. 2012); 3D data was visualized using PyMol (Delano 2004).

\section{Theory of drift correction error analysis}

An accurate metric for testing the quality of image registration with fiducial markers is the target registration error (TRE) (Fitzpatrick and West 2001, Maurer et al. 1997). Here, we adopted the TRE approach to quantify the quality of drift correction. In a first step, the coordinates from $N$ fiducial markers were separated from the coordinates of the fluorophore-labeled biomolecules. Next, the set of trajectories of fiducial markers was separated into two subpopulations, where the first subpopulation $x_{1}$ contained the trajectory of one fiducial marker, and subpopulation $x_{2}$ contained the trajectories of all other fiducial markers. A correction matrix was calculated from $x_{2}$, and $x_{1}$ was transformed with that matrix for every point in time, $t$ (n.b.: $x_{1}$ did not contribute to its own transformation matrix). To calculate the error, the difference between the transformed trajectory, $f_{\text {trans }}$ $\left\{x_{1}\right\}$., and the local weighted mean $f_{L W M}\left\{x_{1}\right\}$ from $f_{\text {trans }}$ $\left\{x_{1}\right\}$ over time, was calculated. This procedure was repeated for all $N$ fiducial markers, and for all frames, $\mathrm{T}$, according to:

$$
\operatorname{TRE}=\sqrt{\left(\frac{1}{N T} \sum_{n=1}^{N} \sum_{t=1}^{T}\left(f_{n, t, t \text { trans }}\left\{x_{n, t, 1}\right\}-f_{n, L W M}\left\{x_{n, 1}\right\}\right)^{2}\right)}
$$

\section{Theory of image registration error analysis}

The 3D coordinate-based colocalization (CBC) algorithm (Malkusch et al. 2012) was used to quantify the error of the registration of different channels (offset correction). For this purpose, we calculated the distribution of single fluorophores around a center molecule using an all-distances function related to Ripley's K-function (Ripley 1977). We calculated the distribution of (i) single molecules detected in channel one around the center molecule and of (ii) single molecules detected in channel two around the same center molecule. The two distributions were correlated (Spearman) and weighted, yielding a $\mathrm{CBC}$ value for the center molecule which can serve as a measure of colocalization with neighboring molecules. This procedure was repeated for every single molecule detected in the image. We applied this approach to fiducial markers to estimate the quality of the image registration (fiducial markers which were used for quality control were not used to calculate the registration matrix).

\section{Results and discussion}

\section{Distribution of fiducial markers in 3D}

Fiducial markers were frequently used for drift correction and image registration in single-molecule localization microscopy (Betzig et al. 2006, Churchman et al. 2005, Rust et al. 2006, Shtengel et al. 2009). Typically, the markers were added to the sample and distributed on the glass surface. This approach often limits their usefulness when imaging away from the surface. Other approaches involved attaching fiducial markers to a cell (e.g. membrane), which in turn can affect singlemolecule detection in the area of the fiducial marker. For drift correction and image registration, fiducial markers should be available in every imaging plane, and should not be attached to the target structure to avoid artifacts in single-molecule localization. Here, we employed a hydrophilic extracellular matrix (ECM), which is commonly used in cell culture (Kleinman and Martin 2005), for super-resolution microscopy. We added the fiducial markers to the ECM gel, embedded the specimen in the matrix and observed the distribution of the markers in 3D (Figure 1).

For drift correction in super-resolution localization microscopy, the spatial position of fiducial markers had to be particularly stable within the gel matrix for the whole acquisition time. We verified this by tracking the

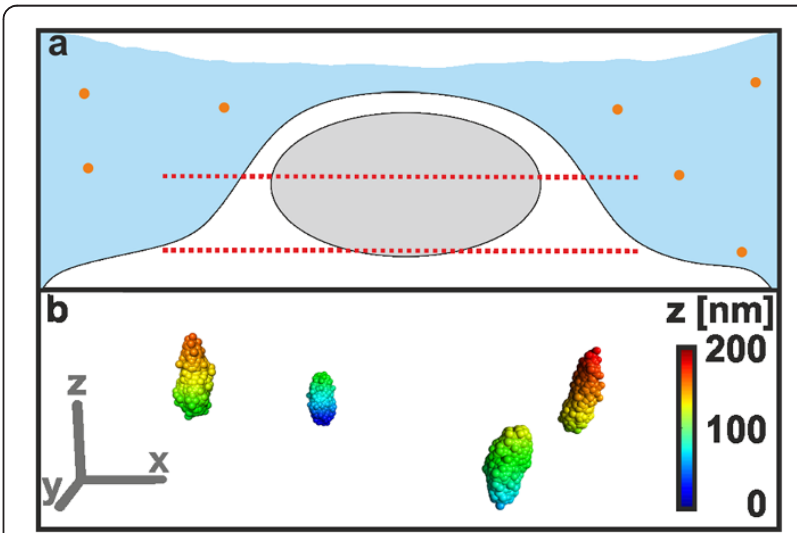

Figure 1 Sample embedding in hydrophilic matrix gel. (a) Fiducial markers can be distributed in an ECM gel in 3D space, and can be used for correction of drift and channel offsets in superresolution microscopy. The distribution in 3D allows recording images in different focal planes; a sufficient density of the same markers in two imaging planes allows 3D registration of superresolution images. (b) The positions of four fiducial markers distributed in the ECM gel were tracked in 3D for an acquisition of 10.000 frames, and are color-coded for the axial position. After drift correction, a localization precision of $6.9 \mathrm{~nm}$ (s.d., lateral) and 18.1 $\mathrm{nm}$ (axial) was determined (scale bars $100 \mathrm{~nm}$ ). 
trajectory of single fiducial markers with respect to the trajectories of the other markers. For that purpose, the distance of a specific fiducial marker to the center of mass of the remaining fiducial markers was calculated. This procedure was repeated for every fiducial marker, and allowed us identifying and removing those that showed an independent movement with respect to the other fiducial markers. In a representative experiment with 33 fiducial markers, we found two which matched these criteria and removed them from further analysis (Additional file 3: Figure S3).

\section{Non-linear drift correction}

In contrast to diffraction-limited microscopy, super-resolution images even suffer from drift in the nanometer range which reduces the spatial resolution (Mlodzianoski et al. 2011). Drift often comprises several non-linear components (Adler and Pagakis 2003). Linear drift correction is not suitable, and more sophisticated methods are needed, for example by using affine or non-linear matrices (see Materials and Methods, Additional file 2: Figure S2) (Betzig et al. 2006, Malkusch et al. 2012).

To first demonstrate drift correction we prepared a sample of fiducial markers immobilized on a glass surface, which was imaged under normal conditions and showed a high level of non-linear drift (an exemplary trajectory is shown in Additional file 2: Figure S2a). From the coordinates of the fiducial markers over time, trajectories were generated which contained information on the movement of the markers. These trajectories were split into spatial dimensions (Additional file 2: Figure S2b) and were convolved with a Gaussian low pass filter (Additional file 2: Figure S2c and S2d). From these trajectories, an affine transfer matrix was calculated for each frame and applied to the uncorrected coordinates of fiducial markers (Additional file 2: Figure S2e). In a second experiment, we embedded fiducial markers in the gel matrix in 3D. Lateral drift was described as above (Additional file 4: Figure S4), axial drift was controlled actively by hardware (see Methods).

\section{Multi-channel image registration}

Imaging more than one spectral channel, focal plane or time point always leads to a spatial offset. A correction of this offset requires multi-channel image registration. To test the suitability of fiducial markers embedded in ECM gel for multi-channel image registration, we prepared a sample in which we visualized three fiducial markers. The sample was imaged in one color, and the image stack was split in two halves. From the two resulting image stacks, two uncorrected super-resolution images were reconstructed (Figure 2a). In the absence of drift, these images should show a colocalization near $100 \%$. By applying drift correction (Figure 2b) and

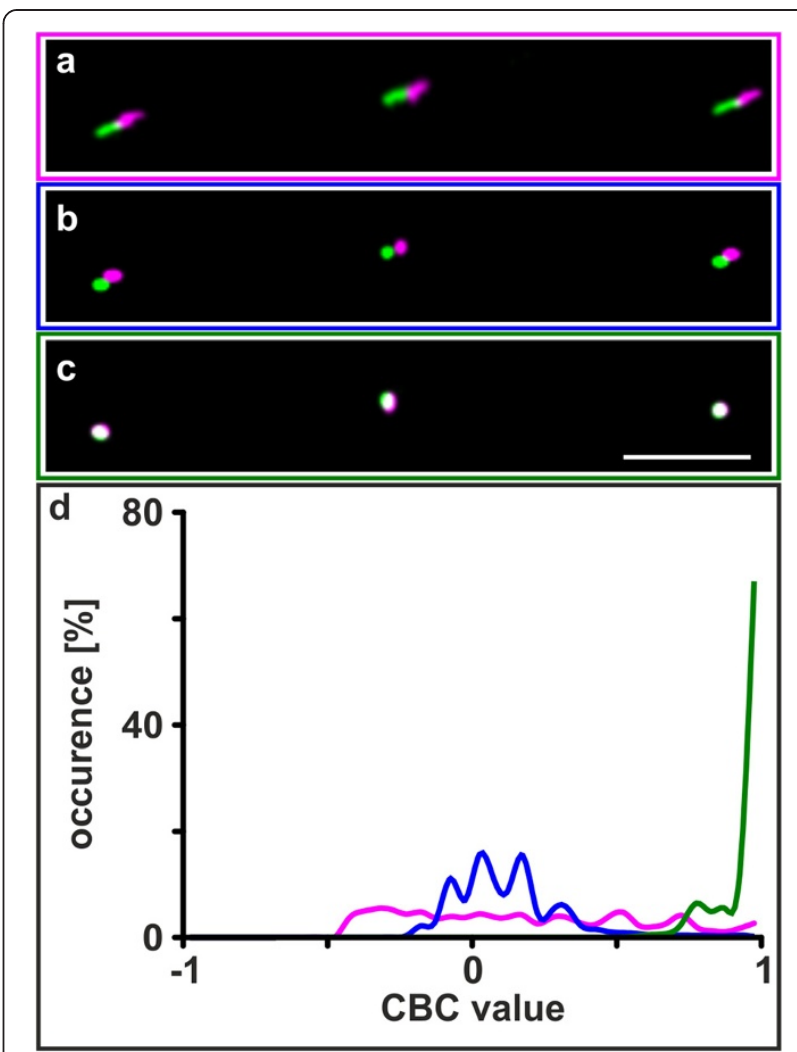

Figure 2 Drift correction and registration of two channels. Fiducial markers were distributed in the ECM gel, imaged and their positions detected: (a) uncorrected super-resolution images, (b) after drift correction and (c) after image registration (scale bars $500 \mathrm{~nm}$ ) The target registration error was determined to $25 \mathrm{~nm}$. (d) The quality of the registered images in (c) is quantified by coordinate-based colocalization (CBC) analysis. The uncorrected (magenta) and the drift-corrected (blue) super-resolution images show no colocalization, whereas the image corrected for drift and temporal offset (green) shows high colocalization.

channel registration (Figure 2c), the mean $C B C$ value is approaching 1 (representing perfect colocalization) (Figure $2 \mathrm{~d}$ ). In contrast, the uncorrected images showed a near uniform distribution of $\mathrm{CBC}$ values. The $\mathrm{CBC}$ values of the drift corrected, but unregistered images on the other hand showed an expected distribution around 0 (representing no colocalization) caused by the spatial offset (Figure 2d). A target registration error analysis for the registration of the two spectral channels yielded a mean registration error of $25 \mathrm{~nm}$ (see Methods).

HeLa cells were labeled for DNA (15 minutes pulse) with both Alexa Fluor 488 and Alexa Fluor 647, and imaged with super-resolution microscopy (Figure 3). Before drift correction and image registration, the fluorescence signal in the two spectral channels showed little overlap (Figures 3a, 3c). After drift-correction and image registration, the spatial pattern of the fluorescence signal recorded for DNA was largely similar and overlapping (Figures 3b, 3d). 


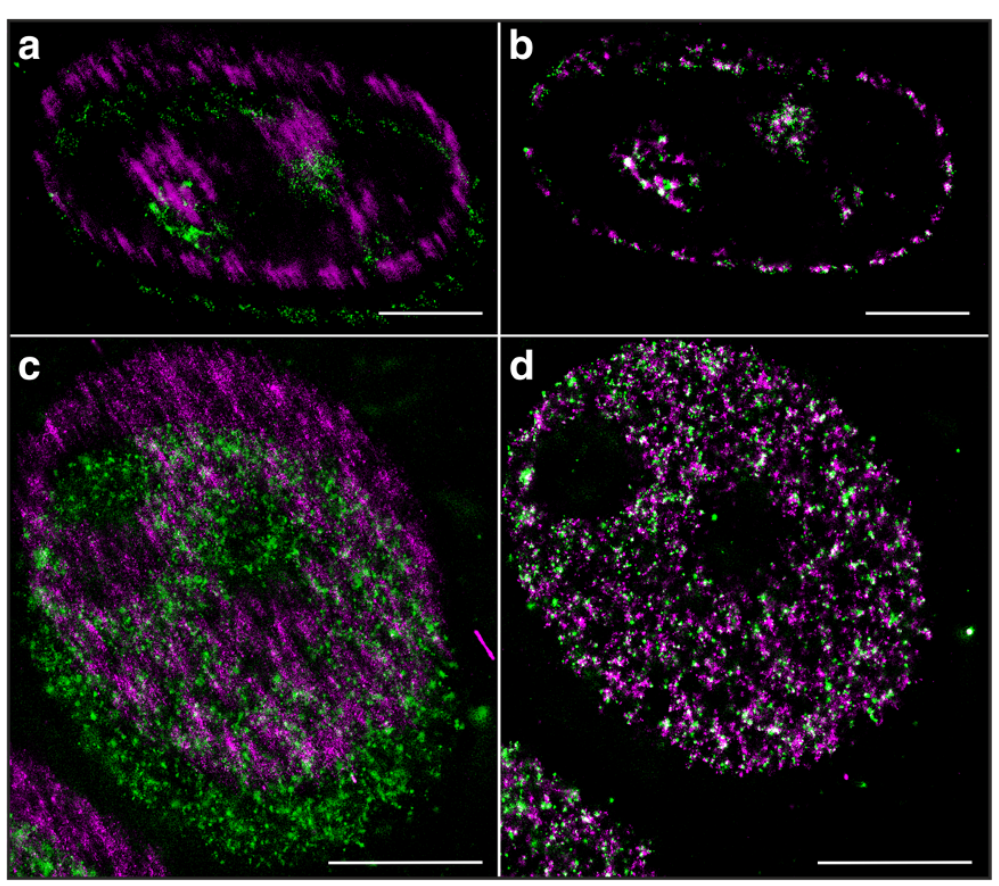

Figure 3 Drift correction and registration of super-resolution images of cells. Cells were treated with the thymidine analogue EdU for 15 minutes, DNA was labeled with both Alexa Fluor 647 (magenta) and Alexa Fluor 488 (green) using click chemistry. HeLa cells were imaged in mid stage $(\mathbf{a}, \mathbf{b})$ and early stage $(\mathbf{c}, \mathbf{d})$, both original images $(\mathbf{a}, \mathbf{c})$ and corrected images $(\mathbf{b}, \mathbf{d})$ are shown (drift and spectral offset was increased for demonstration purpose in $(\mathbf{a}, \mathbf{c})$ ). The corrected images $(\mathbf{b}, \mathbf{d})$ show colocalization of DNA labeled with two fluorophores (scale bars $5 \mu$ m).

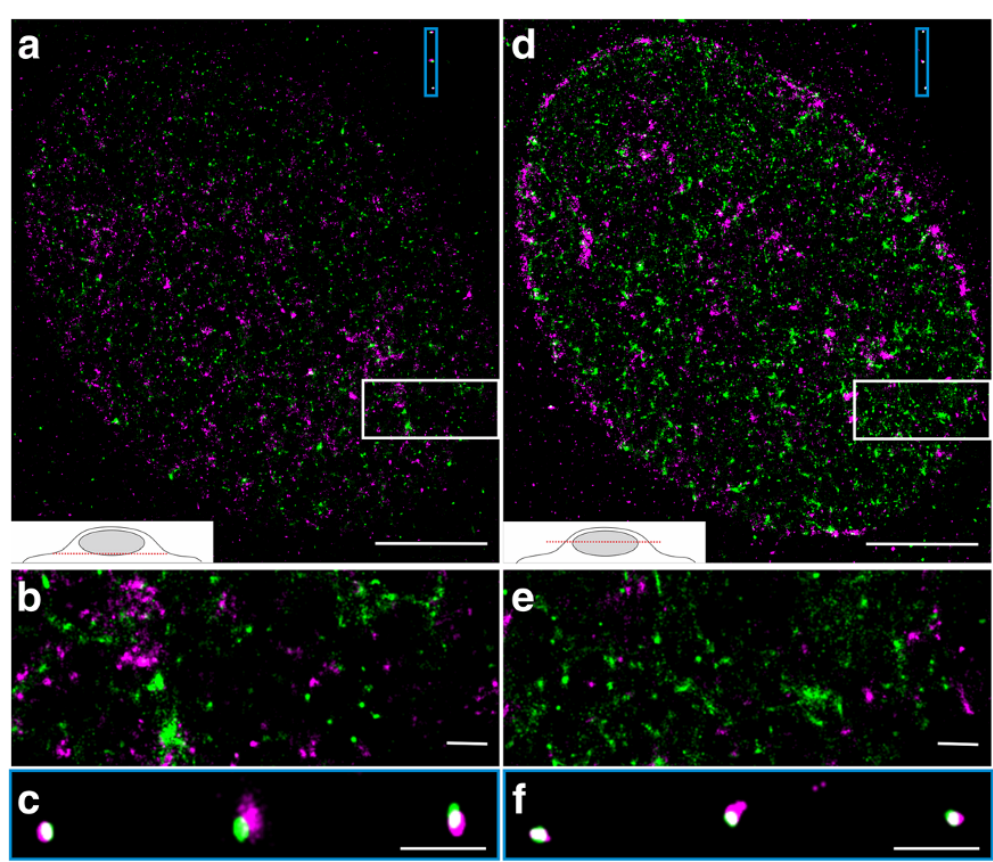

Figure 4 Super-resolution imaging of histone H2B and nascent DNA at different axial planes. Replicated DNA (EdU-Alexa Fluor 647, magenta) and $\mathrm{H} 2 \mathrm{~B}$ (mEos2-H2B, green) were imaged (a) at the bottom and (d) at the middle of a HeLa cell. The nascent DNA shows the typical distribution for a mid S phase nucleus. A magnified view of selected regions (white boxes, $(\mathbf{b}, \mathbf{e})$ ) highlights that the distribution of DNA and H2B is different at this stage of the cell cycle. (c, f) Fiducial markers which were not used for correction of drift and chromatic offset are colocalized and demonstrate the quality of image registration (scale bars $5 \mu \mathrm{m}(\mathbf{a}, \mathbf{d}), 500 \mathrm{~nm}(\mathbf{b}, \mathbf{c}, \mathbf{e}, \mathbf{f})$ ). 


\section{Influence of illumination on image registration}

A possible error source in multi-channel image registration is the use of TIRF or HILO excitation. Both methods are well suited for single-molecule localization microscopy due to the increase in signal-to-noise ratio by background reduction. Still, with both methods, experiments might suffer from non-uniform illumination and light scattering, which get more prominent when imaging with multiple channels deep inside the sample (Oheim and Schapper 2005, Rohrbach 2000, van 't Hoff et al., 2008). Multichannel imaging with super-resolution microscopy benefits from homogeneous TIRF and HILO illumination by scanning the focused laser spot in a circular orbit on the back focal plane (van 't Hoff et al., 2008). Another approach is light sheet illumination, which is decoupling the illumination pathway from the detection pathway (Gao et al. 2012, Keller et al. 2008, Planchon et al. 2011).

\section{D imaging of histone H2B and chromosomal DNA}

Super-resolution images of the histone protein H2B (labeled with the fluorescent protein mEos2) and chromosomal DNA (labeled with Alexa Fluor 647) were recorded at two different axial focal planes of the cell nucleus (Figure 4 and Additional file 5: Figure S5), each providing a 3D section with an axial range of about $1 \mu \mathrm{m}$. Notably, we observed similar photoactivation (mEos2) and photoswitching (Alexa Fluor 647) properties as in aqueous buffers. The super-resolution images were corrected for drift and registered (Figure 4). The spatial pattern of DNA matched typical patterns for the mid (Figure $4 \mathrm{a}, \mathrm{b}$ ) and early (Figure 4c, d) S phase of replication (Nakayasu and Berezney 1989, Zessin et al. 2012). We note that we observe a higher registration error at the limits of the depth of field, which might be due to aberrations (see for example Figure 4c, fiducial marker in the middle, and Additional file 6: Figure S6).

For the generation of a 3D super-resolution image from two 3D sections, we selected fiducial markers in the overlapping space between both planes $(300 \mathrm{~nm}$ ) (Additional file 6: Figure S6). This resulted in a 3D image with an axial range of $1.7 \mu \mathrm{m}$. The acquisition of more 3D sections will further increase the axial range, and whole cells can be visualized. The quality of drift correction and image registration is visualized by 3 fiducial markers at the upper right of the image (Figure $4 \mathrm{a}, \mathrm{d}$ and Additional file 6 : Figure S6). These fiducial markers were not part of the drift correction and registration matrix.

\section{Conclusions}

We introduce a hydrophilic gel matrix for singlemolecule super-resolution microscopy. This matrix matches the index of refraction of aqueous buffers (1.34), and is compatible with photoswitching of synthetic fluorophores in appropriate switching buffers. We demonstrate that single-molecule super-resolution microscopy can be performed in the ECM gel, and that fiducial markers can be added to the ECM gel prior to sample embedding. These fiducial markers are distributed in 3D in the gel matrix, and can be used to correct for drift and image registration. The fiducial markers can be used for imaging away from the cover glass. In addition, this approach allows registration of images recorded in different axial planes, making use of fiducial markers that were recorded in multiple planes. In the future, this approach can be combined with a light sheet illumination scheme and a piezo-driven scanning mode, to allow for automated whole-cell imaging in 3D.

\section{Additional files}

Additional file 1: Figure S1. Sample preparation and data processing. (a) Fiducial markers are added to the hydrophilic gel matrix (extracellular matrix (ECM)) and applied to the specimen. (b) Drift correction and image registration workflow. (TIFF $593 \mathrm{~kb}$ )

Additional file 2: Figure S2. Non-linear drift correction in 2D. We demonstrate the robustness of drift correction with a sample containing 13 fiducial markers which exhibit non-linear drift. (a) Coordinates (before (black) and after (red) drift correction) and calculated centre (triangle, before (blue) and after (magenta) drift correction) of a single fiducial marker. (b) Trajectories (coordinates of fiducial markers over time) were split in $\mathrm{x}$ and $\mathrm{y}$ and (c) convolved with a Gaussian low pass filter to eliminate high frequencies. (d) Smoothed trajectories of 12 fiducial markers were used to calculate the transfer matrix and applied to correct for the non-linear drift of the selected fiducial marker shown in (a) (the fiducial marker in (a) was not used to generate the transfer matrix). This procedure was repeated for all 13 fiducial markers. (e) Distance distribution (left) from all fiducial marker coordinates to its center shown in (a) before (black) and after (red) drift correction. The peak of the distance distribution, which is a measure of the quality of drift control, moved from $40.1 \mathrm{~nm}$ (s.d., uncorrected) to $6.2 \mathrm{~nm}$ (corrected) (respective values for each dimension: $23.5 \mathrm{~nm}$ (before) and $4.5 \mathrm{~nm}$ (after drift correction) in $x$-direction, $32.5 \mathrm{~nm}$ and $4.2 \mathrm{~nm}$ in $\mathrm{y}$-direction). A further and different measure for the quality of drift correction is the covariance matrix (right, distribution of covariances for $x$ (dashed line) and $y$ (straight line) for both uncorrected (black) and corrected (red) coordinates of all fiducial markers). The average covariance decreases from $360.7 \mathrm{~nm}^{2}$ (x, uncorrected) and $698 \mathrm{~nm}^{2}$ (y, uncorrected) to $31.2 \mathrm{~nm}^{2}$ and $24.8 \mathrm{~nm}^{2}$ (corrected).

Additional file 3: Figure S3. Stability of fiducial markers in gel matrix. The stability of fiducial markers $(N=31)$ in the gel matrix was measured over 10.000 frames. The distance variation of a single fiducial marker with respect to the centre of gravity of all other fiducial markers (i.e. 30) over time was calculated. This procedure was repeated for each marker and histogrammed over time, which results in the distributions shown for $x$ (top), $y$ (middle) and $z$ (bottom) (the centre position was set to zero for better comparison). The histograms (right) show the distance variation summed over all frames. The histograms were approximated with a Gaussian function and yielded an s.d. of $3.5 \mathrm{~nm}(\mathbf{x}), 4.9 \mathrm{~nm}(\mathrm{y})$ and 11.3 $\mathrm{nm}(\mathbf{z})$.

Additional file 4: Figure S4. Drift correction of single fiducial markers. Coordinates of four fiducial markers obtained from one super-resolution image (x (red), y (blue) and $\mathbf{z}$ (green)) are shown before (left panel) and after (right panel) drift correction. Drift in $\mathrm{x}$ and $\mathrm{y}$ was corrected as described, drift in z was corrected by hardware stabilization (for details, see Materials and Methods).

Additional file 5: Figure S5. Super-resolution imaging of DNA and histone H2B. Replicated DNA (EdU-Alexa Fluor 647, magenta) and H2B (mEos2-H2B, green) were imaged (a) at the bottom and (b) at the 
middle of a HeLa cell. Drift was not corrected here (see Figure 4 for drift corrected images) (scale bar $5 \mu \mathrm{m}$ ).

Additional file 6: Figure S6. Registration of two 3D stacks. A HeLa cell stained for DNA (EdU-Alexa Fluor 647) was imaged at two different planes (see Additional file 5: figure S5) using 3D super-resolution localization microscopy. The two sets of 3D images were registered by using fiducial markers present in both imaging planes (highlighted in boxes, top right), generating a new 3D stack which spans $1.7 \mu \mathrm{m}$ along the axial direction (scale bar $5 \mu \mathrm{m}$, color coding for c coordinate).

\section{Competing interests}

The authors declare no competing interests.

\section{Authors' contributions}

PJMZ, CLK, SM, UE and MH designed experiments. PJMZ, UE and CLK acquired data. SM, UE and $\mathrm{MH}$ designed the software and analyzed the data. All authors analyzed the data, designed figures and wrote the manuscript. All authors read and approved the final manuscript.

\section{Acknowledgements}

We thank Sven Proppert and Daniela Wengler for their support with initial experiments. The authors gratefully acknowledge financial support by the BMBF (FORSYS, research grant 0315262), the DFG (EXC 115) and the Goethe-University Frankfurt.

Received: 13 March 2013 Accepted: 29 August 2013

Published: 10 September 2013

\section{References}

Adler J, Pagakis SN (2003) Reducing image distortions due to temperaturerelated microscope stage drift. J Microsc 210:131-137

Augst AD, Kong HJ, Mooney DJ (2006) Alginate hydrogels as biomaterials. Macromol Biosci 6:623-633

Bell E, Ivarsson B, Merrill C (1979) Production of a tissue-like structure by contraction of collagen lattices by human fibroblasts of different proliferative potential in vitro. Proc Natl Acad Sci USA 76:1274-1278

Betzig E, Patterson GH, Sougrat R, Lindwasser OW, Olenych S, Bonifacino JS, Davidson MW, Lippincott-Schwartz J, Hess HF (2006) Imaging intracellular fluorescent proteins at nanometer resolution. Science 313:1642-1645

Blomback B, Bark N (2004) Fibrinopeptides and fibrin gel structure. Biophys Chem 112:147-151

Carter AR, King GM, Ulrich TA, Halsey W, Alchenberger D, Perkins TT (2007) Stabilization of an optical microscope to $0.1 \mathrm{~nm}$ in three dimensions. Appl Opt 46:421-427

Churchman LS, Okten Z, Rock RS, Dawson JF, Spudich JA (2005) Single molecule high-resolution colocalization of Cy3 and Cy5 attached to macromolecules measures intramolecular distances through time. Proc Natl Acad Sci USA 102:1419-1423

Delano WL (2004) Use of PYMOL as a communcations tool for molecular science. Abstr Pap Am Chem Soc 228:U313-U314

Endesfelder U, Malkusch S, Flottmann B, Mondry J, Liguzinski P, Verveer PJ, Heilemann M (2011) Chemically induced photoswitching of fluorescent probes-a general concept for super-resolution microscopy. Molecules 16:3106-3118

Endesfelder U, Finan K, Holden SJ, Cook PR, Kapanidis AN, Heilemann M (2013) Multiscale Spatial Organization of RNA Polymerase in Escherichia coli. Biophys J 105:172-181

Fitzpatrick JM, West JB (2001) The distribution of target registration error in rigidbody point-based registration. IEEE Trans Med Imaging 20:917-927

Folling J, Bossi M, Bock H, Medda R, Wurm CA, Hein B, Jakobs S, Eggeling C, Hell SW (2008) Fluorescence nanoscopy by ground-state depletion and single-molecule return. Nat Methods 5:943-945

Galbraith CG, Galbraith JA (2011) Super-resolution microscopy at a glance. J Cell Sci 124:1607-1611

Gao L, Shao L, Higgins CD, Poulton JS, Peifer M, Davidson MW, Wu X, goldstein B, Betzig E (2012) Noninvasive imaging beyond the diffraction limit of 3D dynamics in thickly fluorescent specimens. Cell 151:1370-1385

Heilemann M (2010) Fluorescence microscopy beyond the diffraction limit. J Biotechnol 149:243-251
Heilemann M, van de Linde S, Schuttpelz M, Kasper R, Seefeldt B, Mukherjee A, Tinnefeld P, Sauer M (2008) Subdiffraction-resolution fluorescence imaging with conventional fluorescent probes. Angew Chem Int Ed Engl 47:6172-6176 Hell SW (2009) Microscopy and its focal switch. Nat Methods 6:24-32

Hess ST, Girirajan TP, Mason MD (2006) Ultra-high resolution imaging by fluorescence photoactivation localization microscopy. Biophys J 91:4258-4272

Huang B, Wang W, Bates M, Zhuang X (2008) Three-dimensional super-resolution imaging by stochastic optical reconstruction microscopy. Science 319:810-813

Keller PJ, Schmidt AD, Wittbrodt J, Stelzer EH (2008) Reconstruction of zebrafish early embryonic development by scanned light sheet microscopy. Science 322:1065-1069

Kleinman HK, Martin GR (2005) Matrigel: basement membrane matrix with biological activity. Semin Cancer Biol 15:378-386

Lee J, Cuddihy MJ, Kotov NA (2008) Three-dimensional cell culture matrices: state of the art. Tissue Eng Part B Rev 14:61-86

Malkusch S, Endesfelder U, Mondry J, Gelleri M, Verveer PJ, Heilemann M (2012) Coordinate-based colocalization analysis of single-molecule localization microscopy data. Histochem Cell Biol 137:1-10

Maurer Cr JR, Fitzpatrick JM, Wang MY, Galloway RLJR, Maciunas RJ, Allen GS (1997) Registration of head volume images using implantable fiducial markers. IEEE Trans Med Imaging 16:447-462

Mcgorthy R, Kamiyama D, Huang B (2013) Active microscope stabilization in three dimensions using image correlation. Optical Nanoscopy 2:2-3

Mennella V, Keszthelyi B, Mcdonald KL, Chhun B, Kan F, Rogers GC, Huang B, Agard DA (2012) Subdiffraction-resolution fluorescence microscopy reveals a domain of the centrosome critical for pericentriolar material organization. Nat Cell Biol 14:1159-1168

Mlodzianoski MJ, Schreiner JM, Callahan SP, Smolkova K, Dlaskova A, Santorova J, Jezek P, Bewersdorf J (2011) Sample drift correction in 3D fluorescence photoactivation localization microscopy. Opt Express 19:15009-15019

Nakayasu H, Berezney R (1989) Mapping replicational sites in the eucaryotic cell nucleus. J Cell Biol 108:1-11

Oheim M, Schapper F (2005) Non-linear evanescent-field imaging. J Phys D-Appl Phys 38:R185-R197

Pertsinidis A, Zhang Y, Chu S (2010) Subnanometre single-molecule localization, registration and distance measurements. Nature 466:647-651

Peterson P (2009) F2PY: a tool for connection Fortran and Python programs J Comput Sci Eng 4:296-305

Planchon TA, Gao L, Milkie DE, Davidson MW, Galbraith JA, Galbraith CG, Betzig E (2011) Rapid three-dimensional isotropic imaging of living cells using Bessel beam plane illumination. Nat Methods 8:417-423

Qu D, Wang G, Wang Z, Zhou L, Chi W, Cong S, Ren X, Liang P, Zhang B (2011) 5-Ethynyl-2'-deoxycytidine as a new agent for DNA labeling: detection of proliferating cells. Anal Biochem 417:112-121

Quan T, Li P, Long F, Zeng S, Luo Q, Hedde PN, Nienhaus GU, Huang ZL (2010) Ultra-fast, high-precision image analysis for localization-based super resolution microscopy. Opt Express 18:11867-11876

Ripley BD (1977) Modeling Spatial Patterns. J R Stat Soc Ser B-Methodological 39:172-212

Rohrbach A (2000) Observing secretory granules with a multiangle evanescent wave microscope. Biophys J 78:2641-2654

Rust MJ, Bates M, Zhuang X (2006) Sub-diffraction-limit imaging by stochastic optical reconstruction microscopy (STORM). Nat Methods 3:793-795

Schindelin J, Arganda-Carreras I, Frise E, Kaynig V, Longair M, Pietzsch T, Preibisch S, Rueden C, Saalfeld S, Schmid B, Tinevez JY, White DJ, Hartenstein V, Eliceiri K, Tomancak P, Cardona A (2012) Fiji: an open-source platform for biologicalimage analysis. Nat Methods 9:676-682

Shtengel G, Galbraith JA, Galbraith CG, Lippincott-Schwartz J, Gillette JM, Manley S, Sougrat R, Waterman CM, Kanchanawong P, Davidson MW, Fetter RD, Hess HF (2009) Interferometric fluorescent super-resolution microscopy resolves 3D cellular ultrastructure. Proc Natl Acad Sci USA 106:3125-3130

Tokunaga M, Imamoto N, Sakata-Sogawa K (2008) Highly inclined thin illumination enables clear single-molecule imaging in cells. Nat Methods 5:159-161

van de Linde S, Loschberger A, Klein T, Heidbreder M, Wolter S, Heilemann M, Sauer M (2011) Direct stochastic optical reconstruction microscopy with standard fluorescent probes. Nat Protoc 6:991-1009

Van T, Hoff M, De Sars V, Oheim M (2008) A programmable light engine for quantitative single molecule TIRF and HILO imaging. Opt Express 16:18495-18504 
Wolter S, Schüttpelz M, Tscherepanow M, Van de Linde S, Heilemann M, Sauer M (2010) Real-time computation of subdiffraction-resolution fluorescence images. J Microsc 237:12-22

Xu K, Babcock HP, Zhuang X (2012) Dual-objective STORM reveals threedimensional filament organization in the actin cytoskeleton. Nat Methods 9:185-188

Zessin PJ, Finan K, Heilemann M (2012) Super-resolution fluorescence imaging of chromosomal DNA. J Struct Biol 177:344-348

doi:10.1186/2192-2853-2-4

Cite this article as: Zessin et al: A hydrophilic gel matrix for singlemolecule super-resolution microscopy. Optical Nanoscopy 2013 2:4.

Submit your manuscript to a SpringerOpen ${ }^{\circ}$ journal and benefit from:

- Convenient online submission

- Rigorous peer review

- Immediate publication on acceptance

- Open access: articles freely available online

- High visibility within the field

- Retaining the copyright to your article

Submit your next manuscript at $>$ springeropen.com 